\title{
Motivação metafórica nos phrasal verbs: uma concepção semântico-cognitiva
}

\author{
Samanta Kélly Menoncin Pierozan*
}

\begin{abstract}
Resumo
Este artigo tem como propósito mostrar alguns passos que podem conduzir à identificação das metáforas conceptuais que motivam os sentidos das partículas up e down, elementos constituintes de phrasal verbs, bem como averiguar os mapeamentos metafóricos que motivam essas metáforas, partindo da verificação de quais são os sentidos dessas partículas que expressam os esquemas imagéticos propostos por Rudzka-Ostyn (2003). Este estudo adota como pilar a Teoria da Metáfora Conceptual preconizada por Lakoff e Johnson (1980), uma abordagem semântico-cognitiva, a fim de que se possa refletir sobre a semântica das construções verbo-partícula, cujos significados, na sua maioria, são estendidos metaforicamente. A Linguística de Corpus é utilizada como um recurso metodológico, o que possibilita a realização da coleta de dados autênticos para serem analisados. Pela análise averigua-se que as partículas up e down são utilizadas tanto com sentido prototípico quanto com sentido metafórico, porém, o uso metafórico, também chamado de estendido, prevalece.

Palavras-chave: Phrasal verbs. Semântica Cognitiva. Motivação metafórica. Teoria da Metáfora Conceptual. Linguística de Corpus.
\end{abstract}

\section{INTRODUÇÃO}

Phrasal verbs $(P V s)$ são estruturas geralmente compostas por verbo e preposição, ou verbo e advérbio. Essas construções apresentam sentidos que não são previsíveis a partir do significado da soma de seus componentes. O que ocorre é que, além da contribuição do verbo, as preposições ou advérbios, reconhecidas neste trabalho como "partículas", apresentam forte contribuição semântica para o sentido total dessas construções (verbo-partícula/V-P), motivando um novo significado, o qual geralmente apresenta um forte grau de idiomaticidade.

Com o objetivo de investigar esses significados novos, os quais dizem respeito a extensões metafóricas dos $P V s$, este estudo adota como pilar a Teoria da Metáfora Conceptual (TMC), preconizada por Lakoff e Johnson (1980), uma abordagem * Doutoranda em Letras pelo PDLet - Associação Ampla UCS/UniRitter. Bolsista PROSUP/CAPES. Mestre em Linguística Aplicada pelo PPGLA - UNISINOS. 
semântico-cognitiva. Por meio dessa perspectiva propõe-se refletir sobre a semântica das construções V-P, em especial as que dizem respeito às partículas up e down. Para isso, considera-se a contribuição de Rudzka-Ostyn (2003) a fim de que se possa (i) identificar as metáforas conceptuais que motivam os sentidos das partículas up e down, elementos constituintes dos $P V S$, (ii) averiguar os mapeamentos metafóricos que motivam essas metáforas, e (iii) verificar quais são os sentidos das partículas up e down que expressam os esquemas imagéticos propostos por Rudzka-Ostyn (2003). Para validar essa proposta, a Linguística de Corpus é utilizada como recurso metodológico, possibilitando a extração de dados autênticos, os quais serão analisados.

\section{PRESSUPOSTOS TEÓRICOS}

\subsection{PVs: construções verbo-partícula}

Os $P V s$ são considerados “construções verbo-partícula" (TYLER; EVANS, 2003; LINDNER, 1981; CAPPELLE, 2005), cuja partícula é identificada, por vezes, como um advérbio ou uma preposição. Independentemente da classe gramatical, algumas abordagens teóricas (LINDNER, 1981; CAPPELLE, 2005; TYLER; EVANS, 2003) estabelecem que essa partícula, assim como o verbo, contribui para o significado total do $P V$, produzindo, junto ao verbo, um novo significado, ou mais de um, já que são considerados estruturas complexas. Por esse motivo, os $P V s$ distinguem-se dos chamados verbos preposicionados, os quais, apesar de também serem constituídos de verbo e preposição, não formam um novo significado.

A noção de verbo complexo é utilizada por Lindner (1981) - acredita-se que o motivo que leva a esse tratamento diz respeito às suas estruturas sintática $\mathrm{e}$ semanticamente complexas. De acordo com a autora (1981, p. 2), os $P V s$ podem ser tanto intransitivos, como em (a) The kite floated up (A pipa flutuou) e (b) John fell ill and threw up (John adoeceu e vomitou), quanto transitivos, como em (c) John tossed the cat out before going to bed (John livrou-se do gato antes de ir para a cama) e (d) John ran up a bill (John contraiu uma conta/dívida). Além disso, podem admitir combinações mais literais, como é o caso dos exemplos (a) e (c), constituídos por verbos de movimento e partículas que denotam certo percurso no espaço, ou combinações de elementos mais idiomáticas, cujos constituintes, fora da combinação, não deixam evidente o significado do todo. ${ }^{1}$

1 Ao contrário da língua inglesa, no português, em que os "verbos de movimento" geralmente expressam a noção de direção por meio da raiz, é a própria estrutura verbal que transmite a noção de 
Essas estruturas complexas e indicadoras de idiomaticidade compõem o grupo dos idioms, definido por Kovács e Szabó (1996).

O termo "partícula", frequentemente utilizado ao longo deste trabalho, diz respeito a um dos elementos que constituem os $P V s$. O conceito de partícula é variável, podendo se referir tanto a uma preposição quanto a um advérbio, ou a ambos. Contudo, o papel que a partícula exerce na estrutura do $P V$ é diferente da função de preposições e advérbios, uma vez que contribui com o significado do verbo, formando, junto a ele, um novo significado.

O termo "partícula" é usado para se referir a uma categoria sintática, ou seja, uma "classe de palavra" ou "categoria gramatical" onde faça par com um substantivo, verbo, etc., cujos membros (previsivelmente chamados de "partículas") podem ser identificados por suas possíveis posições no que diz respeito ao verbo e sintagma nominal. (CAPPELLE, 2005, p. 1.)

Segundo Cappelle (2005), o termo "partícula" é usado para se referir a uma categoria sintática, ou seja, uma categoria gramatical. Essa partícula pode ser identificada pela sua possível posição em relação ao verbo e ao $\mathrm{SN}$ objeto. O autor $(2005$, p. 77$)$ argumenta que se deve considerar todas as partículas como uma classe distinta das preposições. Contudo, encontram-se afirmações de que partículas e preposições são similares ou idênticas (CAPELLE, 2005, p. 82).

Quanto ao presente trabalho, não se tem o propósito de discutir se a partícula pertence ou não a uma categoria gramatical própria, mas assume-se que a partícula exerce um papel diferente daquele desempenhado pela preposição, principalmente no que tange às construções V-P, uma vez que se distinguem das combinações verbo-preposição (conhecidos como "verbos preposicionados").

Os $P V s$ também se apresentam em diferentes formatos, os quais são devidos à possível troca de posição de suas partículas. Em suma, a estrutura sintática dos $P V s$ pode variar, podendo ser julgada complexa, pois não se limita a um único padrão sintático, ou mesmo semântico; são combinações que admitem tanto significados literais quanto idiomáticos. Logo, seus sentidos podem ser analisados como tendo diferentes graus de composicionalidade e idiomaticidade, os quais podem ser percebidos por uma escala que, de um lado, é representada por um 
nível mais composicional, de sentidos mais prototípicos e, do outro, um nível mais idiomático, motivando metáforas conceptuais - este último é de especial interesse para a presente investigação.

\subsection{PVs: concepção semântico-cognitiva}

Ao tratar dos fenômenos que os $P V s$ implicam, impreterivelmente remetese à Linguística Cognitiva (LC). Esta, por sua vez, estabelece uma semântica cognitiva, defendendo que a mente é corporificada e que a conceptualização e a razão estão relacionadas a processos imaginativos (metáforas, metonímias, protótipos, espaços mentais, etc.).

De acordo com Lakoff (1987 apud EVANS; GREEN, 2006, p. 157-163), a semântica cognitiva pode ser caracterizada por alguns princípios, dentre eles: (i) a estrutura conceptual é corporificada, ou seja, a natureza da organização conceptual emerge da experiência corpórea; (ii) a estrutura semântica é uma estrutura conceptual, uma vez que é comparada a conceitos; (iii) a representação do significado é enciclopédica, pois unidades linguísticas são vistas como pontos de acesso para o conhecimento em relação a um determinado conceito; e (iv) o significado é construído no nível conceptual, sendo que essa comparação é possível em virtude de um processo dinâmico, em que os itens lexicais são considerados pontos de acesso para o conhecimento e para um conjunto de operações conceptuais.

Conforme Kovács ([2008?], p. 143), os autores Bolinger (1971), Lipka (1972), Sroka (1972) e Fraser (1976) - considerados por ele como gramáticos tradicionais - assumem os $P V S$ como combinações arbitrárias, caracterizados apenas por suas propriedades sintáticas, considerando somente o significado espacial e aspectual da partícula constituinte. Em contrapartida, de acordo com a autora, linguistas cognitivos, como Lindner (1981), Lakoff (1987), Rudzka-Ostyn (2003) e Tyler e Evans (2003), consideram que os significados das partículas de $P V s$ formam uma rede de sentidos relacionados de modo sistêmico. Esses últimos estudiosos também assumem que as estruturas linguísticas são motivadas pelos processos cognitivos - a metaforização é um exemplo. É por esse viés que Lakoff (1987) e Lakoff e Johnson (1980) também afirmam que o sistema conceptual humano é estruturado e definido metaforicamente, já que a organização conceptual é baseada em princípios formados estruturalmente, em que determinados conceitos (os mais 
abstratos) são compreendidos em termos de outros, mais concretos - esse é o tema do próximo subcapítulo, cerne das metáforas conceptuais.

\subsection{A metaforicidade das partículas up e down}

Ao reconhecer que a interpretação dos $P V s$ pode se dar pela abordagem da semântica cognitiva, procura-se investigar os sentidos estendidos e metafóricos das partículas, relacionando-os a esquemas imagéticos. ${ }^{2}$ Para isso, conta-se em especial com as contribuições de Rudzka-Ostyn (2003), abordando os variados sentidos das partículas up e down, tanto em termos de significados prototípicos quanto de significados idiomáticos.

\subsubsection{A partícula UP}

Up é a partícula mais usada na língua inglesa (RUDZKA-OSTYN, 2003, p. 75). Seu uso frequente se explica pela experiência cotidiana (física e abstrata) que se tem em relação a movimentos ou posições ascendentes. A autora apresenta alguns sentidos, estendidos metaforicamente, que partem do sentido mais prototípico da partícula, o qual diz respeito à verticalidade ascendente e é representado por Rudzka-Ostyn através do esquema imagético "posição superior ou mover em direção a um lugar mais alto".

Na sequência, são abordados os sentidos de up apontados pela autora.

Sentido 1 - UP: posição superior ou mover em direção a um lugar mais alto

Tipicamente, o sentido espacial de up representa o movimento de um lugar mais baixo a um mais alto (e.g. Could you go up and see whether the baby is asleep? - Você poderia subir e ver se o bebê está dormindo?). Contudo, a partícula também pode tratar de situações em que a entidade muda de uma posição horizontal para uma vertical (e.g. We all stood up when the president entered - Todos nós

2 De acordo com Evans (2007, p. 106), os "esquemas imagéticos" dizem respeito a representações conceptuais abstratas que emergem da interação direta que se tem com o mundo, derivando das experiências sensoriais e perceptuais, ou seja, da experiência corporificada. Por questões econômicas, as imagens correspondentes a esses esquemas não são ilustradas neste artigo, mas podem ser verificadas em Rudzka-Ostyn (2003). 
nos levantamos quando o presidente entrou). Além disso, pode não haver uma mudança de lugar; isso ocorre quando a posição de dada entidade já está em um nível superior a outras (e.g. Put the bottle up on the shelf - Coloque a garrafa na prateleira).

Sentido 2 -UP (to): visar ou atingir um objetivo, um fim, um limite

A partícula up, além de referir-se a movimento ascendente, também pode indicar movimento em direção ao lugar em que a entidade está ou, pelo menos, deveria estar, principalmente quando ela é combinada com a partícula to - não se trata, necessariamente, de um movimento ascendente/acima. Essas descrições podem ser evidenciadas nos exemplos Several passengers rushed up to the waiting bus (Diversos passageiros foram às pressas para o ponto de ônibus) e On cold wet mornings I cannot start my old car up (Nas manhãs úmidas e frias eu não consigo ligar meu carro velho).

Sentido 3 - Norte é UP, sul é DOWN

Uma vez que o norte está convencionalmente representado no topo da página dos mapas, torna-se comum perceber essa área geográfica como up (e.g. Let's leave London and go up to Edinburgh for the weekend - Vamos sair de Londres e ir para Edimburgo no final de semana), assim como a parte sul é percebida como down.

Sentido 4 - UP: mover para um nível, valor ou medida superior

Referindo-se a domínios abstratos, de modo geral, recebem atributos, os quais são tratados em termos de domínios mais concretos. Essa correspondência de um domínio concreto a um abstrato é possível a partir da noção espacial, que se expressa por meio da noção de verticalidade. Trata-se de domínios que envolvem temperatura, categorias sociais e profissionais, atitudes, conhecimento, opiniões, sentimentos, posse, acessibilidade a coisas, graus de intensidade, etc. Conforme Rudzka-Ostyn (2003, p. 80), o que eleva ou o que é bom, bonito, gratificante, forte, sólido, corajoso ou positivo está no topo da dimensão vertical, ou seja, acima (up), e o que decai/decresce ou o que é ruim, feio, triste, pequeno, fraco, 
frágil, acobardado ou negativo está na base dessa linha vertical de avaliação, isto é, está abaixo (down). Alguns exemplos: Judy's temperature is going up, she may be getting $f u$ (A temperatura de Judy está subindo, ela pode estar pegando uma gripe) - temperatura; I hope to move up to a management position within five years (Eu espero subir para uma posição gerencial dentro de cinco anos) - categoria profissional; At 16, Joe is very mature, he has really grown up very fast (Aos 16, Joe é muito maduro, ele realmente cresceu muito rápido) - atitude; I need to brush up my English (Eu preciso desenferrujar meu inglês) - conhecimento; She cheered up when she saw the presents her friends had brought (Ela se animou quando viu os presentes que seus amigos trouxeram) - sentimento; e As granny is slightly deaf, you have to speak up a bit (Como a vovó é um pouco surda, você tem de falar um pouco mais alto) - grau de intensidade.

Sentido 5 - UP: o que está acima é mais visível, acessível e conhecido

Entidades, concretas ou abstratas, e que estão ou chegam a um nível ou local mais elevado são percebidas mais facilmente, pois chamam mais atenção. Além disso, uma das características de muitos verbos que combinam com a partícula up é a de tornar mais visível e conhecido o que estava omitido ou desconhecido. Como exemplos, pode-se considerar: He was determined to bring the issue up at the meeting (Ele estava determinado a trazer o assunto à tona na reunião) / The mistake in the fabric won't show up once the dress is made (A falha no tecido não aparecerá, uma vez que o vestido estiver feito).

Sentido 6-UP: cobrir uma área completamente/atingir o limite máximo

$U p$, além de indicar o alcance de limites abstratos, também indica que um objeto, no seu todo, pode ser afetado por uma ação. Dessa forma, dizer Cut up the meat! significa cortar toda a carne, em cubos/pedaços, e não apenas parte dela. Na mesma linha, pode-se verificar, na sentença Mary gave up the idea of leaving (Mary desistiu da ideia de partir), que up aponta para um "nível" em que a ideia é considerada desinteressante e, portanto, abandonada" (RUDZKA-OSTYN, 2003, p. 87).

Além disso, a autora afirma ser possível perceber que, às vezes, up expressa chegada a um topo, podendo ser o ponto mais alto de uma trajetória vertical ou a 
fronteira de uma dada localização, etc. Essa noção de topo ou de fronteira pode ser estendida metaforicamente em termos de qualquer limite abstrato.

\subsubsection{A partícula DOWN}

Após up, out e off, down é a partícula mais utilizada (RUDZKA-OSTYN, 2003, p. 104). O movimento e a orientação de down, que se dá tanto no nível concreto quanto no nível abstrato ou metafórico, são comuns às experiências cotidianas. Essa relação de orientação e experiência está associada ao modo como se percebe down - como sendo posição inferior e em oposição à partícula up. Essa orientação baseada na experiência física e cultural ocorre porque o sistema conceptual é controlado pela posição vertical do corpo humano, conforme explicam Lakoff e Johnson (1980, p. 14). O sentido mais prototípico de down é "movimento de um lugar mais alto para um mais baixo" e os demais são extensões desse.

Sentido 1 - DOWN: movimento de um lugar mais alto para um mais baixo

Frequentemente, down indica que um objeto é deslocado de um ponto mais alto para um ponto mais baixo (e.g. Could you go down to the cellar and bring us up a few bottles of beer? - Você poderia descer no porão e nos trazer algumas garrafas de cerveja?). No entanto, nem sempre há mudança da localização em si, mas da posição de um determinado objeto da vertical para a horizontal. Rudzka-Ostyn exemplifica dizendo que uma árvore, quando cortada, tem sua posição modificada, da vertical para a horizontal (e.g. Large trees are hauled down from the mountains and taken to the saw mill - Árvores de grande porte são arrastadas montanhas abaixo e levadas para as serrarias). Além disso, a autora afirma que o uso de down também indica a mudança de posição de apenas parte de uma entidade (e.g. sit down - sentar; kneel down - ajoelhar) e que, quando nenhum movimento ocorre, a entidade é posicionada mais abaixo do que de costume, ou sua posição fica mais na horizontal do que na vertical (e.g. The doctor asked me to strip down for the medical check-up - O médico pediu que eu me despisse para o exame médico).

Sentido 2-DOWN: tempo e movimento orientados geograficamente 
Dois usos metafóricos de down dizem respeito ao tempo e à orientação geográfica de um objeto ou lugar. O tempo, visto como uma superfície, refere-se, com frequência, a um ponto no tempo, posterior a outro ponto (e.g. She will go down in history as the greatest opera singer - Ela será lembrada na história como a melhor cantora de ópera). Em relação à orientação geográfica indicada por down, seu uso aponta para uma entidade que se move para o sul ou está localizada ao sul de um dado lugar (e.g. Let's leave Edinburgh and go down to London - Vamos partir de Edimburgo e ir/descer para Londres).

Sentido 3-DOWN: redução de intensidade, qualidade, quantidade, tamanho, grau, valor, atividade, status, força...

Segundo Rudzka-Ostyn (2003, p. 107), a maioria dos usos espaciais e literais de down indica que uma entidade deslocou-se, ou foi deslocada, de um lugar mais alto para um mais baixo. Essas mudanças no espaço associam-se a diferenças que ocorrem em termos de vários domínios abstratos, como volumes, temperaturas, peso, preços, emoções, senso de importância ou respeito, relações sociais, poder, etc. - cujas associações metafóricas expressam redução de intensidade e valor. Os exemplos a seguir, extraídos de Rudzka-Ostyn, ilustram alguns desses domínios: I don't know why, but I really feel down these days (Eu não sei o porquê, mas eu realmente me sinto para baixo nestes dias) - emoção; Fierce competition brought prices down (Uma competição intensa fez os preços caírem) - preço; The university cut down scholarships by $25 \%$ to save money (A universidade cortou as bolsas de estudo de $25 \%$ para economizar) - volume/quantidade; The government is trying to put down all political opposition (O governo está tentando derrubar todas as oposições políticas) - poder; A true friend never lets you down (Um amigo verdadeiro nunca te deixa cair/pra baixo) - relações sociais; e At the meeting I explained that we had to change, but people talked me down (a reunião eu expliquei que nós tínhamos de mudar, mas o pessoal fez pouco caso de mim) senso de respeito/desrespeito.

Sentido 4 -DOWN: atingir um objetivo, realização, limite em uma escala para baixo 
Uma variedade de verbos combinados com up representa intensidade e completude de ações. Da mesma forma, muitos verbos combinados com down também expressam completude/realização, porém dizem respeito ao nível mais baixo da escala de grau, valor, medida, etc. As sentenças a seguir ilustram esse sentido: (i) Several houses and shops burned down in the devastating fire (Diversas casas e lojas queimaram no incêncio devastador), (ii) Our cat is so old and ill, we'll have to ask the vet to put her down (Nosso gato está tão velho e doente que teremos de pedir ao veterinário para sacrificá-lo) e (iii) I could not finish my article because my computer broke down (Eu não consegui terminar meu artigo porque meu computador quebrou).

Sentido 5 - DOWN: movimentos de comer ou escrever

Conforme Rudzka-Ostyn (2003, p. 112), “considerando o movimento descendente que acompanha alimentação e escrita, não é surpresa que muitos dos verbos que expressam essas atividades contenham a partícula down", como em He was so hungry that he wolfed down a whole chicken (Ele estava tão faminto que devorou um frango inteiro) e She put down every single word uttered by the professor (Ela anotou/criticou todas as palavras proferidas pelo professor). Além disso, a autora afirma que a partícula também pode ser associada não só a verbos, mas a substantivos e advérbios para formar nomes compostos e que constituem novos significados, como downstairs (andar de baixo), downfall (queda) e upside down (ao contrário/de cabeça para baixo).

\section{Metodologia e coleta de dados}

Este estudo adota a Linguística de Corpus como recurso metodológico, a fim de que se possa coletar os dados necessários. Para isso, escolheu-se um corpus autêntico, representativo e adequado à pesquisa - The Corpus of Contemporary American English (COCA).

Para se chegar aos dados a serem analisados, algumas decisões conduziram a coleta de concordâncias, são elas: (i) como primeira etapa de seleção dos $P V s$ para análise, identificar os $100 P V s$, no formato V-P-SN, mais frequentes do corpus da pesquisa, ou seja, os $50 P V s$ mais frequentes para cada partícula; (ii) a partir dessa lista de $P V s$ mais frequentes, verificar, no dicionário especializado Macmillan 
Phrasal Verbs Plus, quais são as três construções com up e as três construções com down que apresentam maior número de acepções; e (iii) na sequência, averiguar quais são as 10 primeiras concordâncias do corpus COCA constituídas pelos seis $P V s$ selecionados, considerando todas as formas de lematização dos verbos para cada agrupamento. Buscam-se e averiguam-se essas sequências de concordâncias por meio da ferramenta KWIC, extraindo-as e armazenando-as em formato ".xlsx". Posteriormente, identificam-se e descartam-se, manualmente, os agrupamentos lexicais que não se classificam como $P V s$.

Observa-se que a escolha do dicionário Macmillan Phrasal Verbs Plus se deu pelo fato de exibir os sentidos dos $P V s$ de maneira interrelacionada, apresentando redes semânticas organizadas a partir da polissemia dos $P V s$. Foram encontrados outros dicionários especializados de $P V s$; contudo, esses materiais exibiam seus sentidos de forma desconexa, dando a entender que a memorização seria a única forma de apreender seus significados.

Uma outra tomada de decisão que se faz necessário justificar diz respeito à quantidade de $P V S$ selecionados - $100 P V s$. Conforme abordado, nem todos os $P V s$ possuem forte grau de idiomaticidade ou são altamente polissêmicos, por essa razão, precisou-se definir uma quantidade de construções que viesse a contemplar diversos $P V s$, em especial aqueles com variadas acepções, o que, consequentemente, no momento da análise, tende a contemplar os sentidos propostos por Rudzka-Ostyn (2003).

Tendo feito a seleção dos $P V s$, bem como a coleta de suas respectivas concordâncias, realizam-se três novas etapas, as quais tratam, especificamente, da análise dos dados; são elas: (i) relacionar o significado dos $P V S$ selecionados com os sentidos e esquemas imagéticos propostos por Rudzka-Ostyn (2003), (ii) averiguar quais conceitos são mapeados de um domínio a outro e (iii) identificar as metáforas conceptuais, em especial aquelas do tipo orientacional, conforme Lakoff e Johnson (1980), que estão vinculadas aos $P V S$ e às respectivas concordâncias coletadas e selecionadas para análise. O resultado dessas etapas está representado no Quadro 1 e no Quadro 2, na seção 3.

\section{Dados e achados}

A partir dos procedimentos metodológicos realizados, fez-se a seleção dos seguintes PVs: pick up, come up, set up, go down, come down e break down. 
Centrando-se nessas construções foi possível chegar às concordâncias que são apresentadas e analisadas na sequência. As concordâncias, dispostas em quadros, foram assim organizadas a partir de alguns critérios tomados.

Nos quadros a seguir, faz-se uso das abreviações "M" para significar "mapeamento" e "MC" para significar "metáfora conceptual". Os achados (metáforas orientacionais e mapeamentos) também estão dispostos nos quadros.

QUADRO 1 - Sistematização das concordâncias constituídas por $P V s$ com $u p$ Sentido 1: posição superior ou mover em direção a um lugar mais alto "\# "But aren't you curious? "Barbara picked up a letter she'd set aside." Listen - this his six-week rotation in psychiatry, for example, "they picked up all the patients here and moved them to Fitzsimons, " be alarmed; he often dozed off after eating. She picked up the tray, put it on the night table, then

heard the invaders? I had forgotten to listen. Lulwa picked up the knife, started chopping again. The onion fell away goddess, and I slept. \# In the morning I picked up my astrology books and began to study. And over every

"Oh, are we there already?" \#Kelly picked up his book bag while the Navatar opened the door for him

And strength only carried him for a few minutes. He picked

up the football and stepped outside, flinging it down the street

it soon, whenever you are ready. "My hands came up to my chest and touched the tiny breasts there. It

Sentido 2: visar ou atingir um objetivo/fim/limite

that evening and haggled the price down for us.

She picked up our mail and delivered it herself, she got free medicine

\begin{tabular}{l|l} 
MC: & CAPTAR/OBTER ESTÁ PARA CIMA \\
\hline M: & verticalidade ascendente/objetivo orientado $\rightarrow$ ato de obter \\
\hline
\end{tabular}

attack on his character, which it wasn't. He came up to me and tried to have a photo opportunity. I

Lake, basking in the good aura, and a couple came up to me a few minutes ago. They were a good-looking

in 2008 for the Dodgers and Padres. \# "I came up here just to say thank you", Maddux said to

\begin{tabular}{l|l|}
\hline MC: & PRÓXIMO ESTÁ PARA CIMA \\
\hline M: & verticalidade ascendente/objetivo orientado $\rightarrow$ ato de aproximar \\
\hline $\begin{array}{l}\text { 'This'll work. Trust me. I've been setting up this joke for six years now. KOTB: All } \\
\text { right. }\end{array}$
\end{tabular}




\begin{tabular}{|c|c|}
\hline MC: & TER CONTROLE/FORÇA ESTÁ PARA CIMA \\
\hline M: & Verticalidade ascendente/objetivo orientado $\rightarrow$ controle organizacional \\
\hline \multicolumn{2}{|c|}{$\begin{array}{l}\text { you can probably do better. I did, by setting up automatic forwarding of all my } \\
\text { persona e-mail to Google 's }\end{array}$} \\
\hline MC: & CRIAR/PREPARAR ESTÁ PARA CIMA \\
\hline M: & Verticalidade ascendente/objetivo orientado $\rightarrow$ ato de criar \\
\hline \multicolumn{2}{|c|}{$\begin{array}{l}\text { Circle of five gold stars on his collar, will be setting up his } \\
\text { command post in accordance with prefire plans at the fire }\end{array}$} \\
\hline MC: & CRIAR/PREPARAR ESTÁ PARA CIMA \\
\hline M: & Verticalidade ascendente/objetivo orientado $\rightarrow$ ato de criar \\
\hline \multicolumn{2}{|c|}{ Sentido 4: mover para um nível/valor/medida superior } \\
\hline \multicolumn{2}{|c|}{$\begin{array}{l}\text { Think that David Stern has moved off that number. He came up to } 52 \text { percent, and the } \\
\text { players union was actually at }\end{array}$} \\
\hline $\mathrm{MC}:$ & MAIS ESTÁ PARA CIMA \\
\hline M: & verticalidade ascendente $\rightarrow$ quantidade/tamanho/intensidade \\
\hline \multicolumn{2}{|c|}{ Sentido 5: o que está acima é mais visível/acessível/conhecido } \\
\hline \multicolumn{2}{|c|}{$\begin{array}{l}\text { our home?" On August 17, 1992, I picked up Hurricane Andrew's image from a } \\
\text { Russian satellite. I tracked }\end{array}$} \\
\hline \multicolumn{2}{|c|}{$\begin{array}{l}\text { "\# Back in his office, Zelman picked up his messages. More congressional aides had } \\
\text { called from }\end{array}$} \\
\hline MC: & CAPTAR/OBTER ESTÁ PARA CIMA \\
\hline M: & verticalidade ascendente/objetivo orientado $\rightarrow$ ato de captar/obter \\
\hline \multicolumn{2}{|c|}{$\begin{array}{l}\text { here. Not surprisingly, the issue of moving the trial came up. Defense attorneys for } \\
\text { Timothy McVeigh and Terry Nichols say }\end{array}$} \\
\hline \multicolumn{2}{|c|}{$\begin{array}{l}\text { then so be it. The SWAT troopers 'heads came up; something was going on, and } \\
\text { they were getting the }\end{array}$} \\
\hline \multicolumn{2}{|c|}{$\begin{array}{l}\text { about it doesn't help the work. When that question came up, I was like, Here we } \\
\text { go. Let's }\end{array}$} \\
\hline MC: & (MAIS) VISÍVEL ESTÁ PARA CIMA \\
\hline M: & Verticalidade ascendente $\rightarrow$ visibilidade/acessibilidade/conhecimento \\
\hline \multicolumn{2}{|c|}{$\begin{array}{l}\text { "while I was talking to my wife, and those came up. " \# Susan loomed in. There } \\
\text { were several about }\end{array}$} \\
\hline \multicolumn{2}{|c|}{$\begin{array}{l}\text { GROSS: Can I as } \\
\text { Prostitute? Mr- }\end{array}$} \\
\hline MC: & EXISTÊNCIA ESTÁ PARA CIMA \\
\hline M: & verticalidade ascendente/objetivo orientado $\rightarrow$ ato de tornar algo possível \\
\hline \# Staf & $\begin{array}{l}\text { When a millionaire gambling magnet (Cleese) sets up a cross- } \\
\text { between a group of contestants, they }\end{array}$ \\
\hline
\end{tabular}


trains pastors to stand in judgment of the texts. This sets up the pastors for a jolt when they find themselves in the

from supplying news to broadcasters,

CBS responded in 1933 by setting up its own news-gathering unit. CBS News reports during World

who take care of him. "She also began setting up a support network of friends and neighbours. The phone

to achieve. He is positive

about Labour's idea of setting up an independent commission to find a consensus on funding

of America, but he is able to keep working by setting up a laptop, a printer and a stapler in his room

\begin{tabular}{|l|l|}
\hline MC: & CRIAR/PREPARAR ESTÁ PARA CIMA \\
\hline M: & Verticalidade ascendente/objetivo orientado $\rightarrow$ ato de criar \\
\hline $\begin{array}{l}\text { Brilliant sunlight floods over the skirt of her dress, setting up a contrast with her head, } \\
\text { which is hidden in shadow }\end{array}$ \\
\hline MC: & (MAIS) VISÍVEL ESTÁ PARA CIMA \\
\hline M: & Verticalidade ascendente $\rightarrow$ visibilidade/acessibilidade/conhecimento \\
\hline
\end{tabular}

Fonte: Elaborado pela autora.

Observa-se no quadro acima que, para o Sentido 1, não há indicação de qualquer mapeamento e de metáfora conceptual. O que ocorre é que se trata de ocorrências não metafóricas, em que o sentido mais prototípico de up é evidenciado; muito provavelmente porque a carga semântica do verbo se sobrepõe em relação ao todo.

No que diz respeito ao Sentido 2, o mesmo foi verificado em sete concordâncias, contemplando os três $P V s$ selecionados. Nessas concordâncias o significado da partícula é estendido metaforicamente para indicar movimento de aproximação de um objetivo ou limite de um domínio abstrato, a fim de que possa ser compreendido como ato de se obter algo desejado, de criar ou de aproximar-se de algo. Trata-se de um sentido que tem forte relação com a noção de verticalidade ascendente.

O Sentido 3 não é apresentado no quadro acima, uma vez que o mesmo não foi contemplado pelos $P V s$ selecionados. Trata-se de uma ocorrência não tão comum (dentre os $P V s$ selecionados). Com relação ao Sentido 4, verificou-se apenas uma ocorrência, a qual tem como nódulo o $P V$ come up. Utilizado para descrever uma entidade abstrata, quantidade, é compreendido em termos de verticalidade ascendente. 
Intimamente relacionado a um nível mais elevado de entidades abstratas, o Sentido 5, contemplado pelos três $P V s$ selecionados, possibilita às entidades maior visibilidade, acessibilidade e/ou que sejam conhecidas. Esse é o sentido mais recorrente; dentre 30 concordâncias com $u p, 14$ delas contemplam esse sentido.

QUADRO 2 - Sistematização das concordâncias constituídas por $P V s$ com down Sentido 1: movimento de um lugar mais alto para um mais baixo Icy (Photo-of-Patrick-o)Mr-V-MARSH: Every time I'd go down a hill that was, you know, fairly steep the sled

school record in the decathlon. "You had to go down about eight stairs to get to the front door. The distance

point the tail must

stop, reverse direction, and go down again. This reversal could be accomplished actively, through

enough in the morning to

realize that I need to go down and get it before they get there.

Coordination and

his cows" getting out

of the fences "and going down and grazing on the banks of the railroad. \# "The

- almost. "Nash, I want you to go down and make sure everything looks good in the engine room; check

listen while his aide talks into the mike, then coming down again and making sure the podium is draped right and the sign

the American Heart Association. And he asked

me to come down and drive the - the environmentally safe beverage cart around

It was sort of an open call for folks to come down and help us out and tell us how we could really make

the hills. "A few days later, residents came down and returned to normal life, thankful that they minded island

Sentido 3: redução de intensidade, qualidade, quantidade, tamanho, grau, valor, atividade, status, força... 
Went up 2.1 percent and

wages, salaries and benefits went down 3 percent. There's a widening gap, as

you explain this guy at United Airlines who- their profits went

down 70 percent- Mr. BRINDISI: That's in one year. That

say there's no single trick to it, but go down a list of improvements and a car's mileage will go up

Weight-wise, she's halfway there and has even gone down a shoe size. But emotionally, she's on the road

on future gasoline prices, are that the price is coming down a little bit; that is, that we'll have a

\begin{tabular}{l|l}
\hline MC: & MENOS ESTÁ PARA BAIXO \\
\hline M: & Verticalidade descendente $\rightarrow$ quantidade/tamanho/intensidade \\
\hline
\end{tabular}

at all. As a matter of fact, he came down and did his traditional "Woo" like he always does upon

\begin{tabular}{l|l}
\hline MC: & CONHECIDO ESTÁ PARA BAIXO \\
\hline M: & Verticalidade descendende $\rightarrow$ conhecimento \\
\hline
\end{tabular}

there,you know. Mr-Robinson: Then the admiral came down and explained to us what our responsibilities were.

\begin{tabular}{|l|l|}
\hline MC: & BAIXO STATUS ESTÁ PARA BAIXO \\
\hline M: & Verticalidade descendente $\rightarrow$ baixo status social \\
\hline $\begin{array}{l}\text { of control, he does a good job. He broke down and cried after that } 1992 \text { loss } \\
\text { to the Redskins even though }\end{array}$ \\
\hline $\begin{array}{l}\text { the midst of his torrent of words, his translator actually broke } \\
\text { down and cried out, "I can't take it anymore }\end{array}$ \\
\hline $\begin{array}{l}\text { Ominously, Lieutenant Koeppen in the Protos was completely broken down } \\
\text { and losing time." I wish we were }\end{array}$ \\
\hline MC: & EMOCIONAL ESTÁ PARA BAIXO \\
\hline M: & Verticalidade descendente $\rightarrow$ sentimento/emoção \\
\hline $\begin{array}{l}\text { the husband of my last roommate, "she says, breaking down and weeping at } \\
\text { the memory. After three months in }\end{array}$ \\
\hline MC: & TRISTE ESTÁ PARA BAIXO \\
\hline
\end{tabular}




\begin{tabular}{|c|c|}
\hline M: & Verticalidade descendente $\rightarrow$ tristeza \\
\hline \multicolumn{2}{|c|}{ Sentido 4: atingir um objetivo, realização, limite em uma escala para baixo } \\
\hline \multicolumn{2}{|c|}{$\begin{array}{l}\text { people who just ignored the bureaucracy and just decided to come } \\
\text { down and do what you could, even though no one-there }\end{array}$} \\
\hline MC: & OPOSIÇÃO ESTÁ PARA BAIXO \\
\hline M: & Verticalidade descendente $\rightarrow$ ato de opor-se a algo ou alguém \\
\hline \multicolumn{2}{|c|}{$\begin{array}{l}\text { chase down a suspect? Wrestle him to the ground? Break down a door? Hit } \\
\text { the deck, roll, draw your }\end{array}$} \\
\hline \multicolumn{2}{|c|}{$\begin{array}{l}\text { two volunteers went to the } \\
\text { food vendor's tent and broke down a large pile of corrugated } \\
\text { cardboard for loading in a nearby }\end{array}$} \\
\hline \multicolumn{2}{|c|}{$\begin{array}{l}\text { I was able to learn the rules of acting. You break down a script. You don't } \\
\text { walk unless you have a }\end{array}$} \\
\hline \multicolumn{2}{|c|}{$\begin{array}{l}\text { Made a decision. He was } \\
\text { convinced that his marriage had broken down and he was going to ask } \\
\text { his wife for a divorce }\end{array}$} \\
\hline \multicolumn{2}{|c|}{$\begin{array}{l}\text { a result, they are more } \\
\text { difficult for the body to break down at night, because the metabolism has } \\
\text { slowed for sleep. }\end{array}$} \\
\hline \multicolumn{2}{|c|}{$\begin{array}{l}\text { would continue to flourish precisely } \\
\text { because it had begun to break down barriers. \# "The problem with } \\
\text { college athletics is the }\end{array}$} \\
\hline MC: & DESINTEGRAÇÃO ESTÁ PARA BAIXO \\
\hline M: & $\begin{array}{l}\text { Verticalidade descendente/objetivo orientado } \rightarrow \text { ato de desintegrar/ } \\
\text { quebrar/derrubar }\end{array}$ \\
\hline
\end{tabular}

Fonte: Elaborado pela autora.

Nota-se que, no quadro acima, não há indicação de mapeamento para o Sentido 1, uma vez que o mesmo diz respeito ao sentido mais prototípico da partícula e, portanto, pertence a um nível mais composicional da construção. No que diz respeito ao Sentido 3, verifica-se que a MC é motivada pela projeção de verticalidade descendente dos domínios quantidade, tamanho e intensidade, assim como conhecimento, baixo status social e tristeza. 
Quanto ao Sentido 4, todos os casos verificados são fundamentados conceptualmente na noção de objetivo orientado e descendente. Para esse sentido, os conceitos de oposição e de desintegração foram os motivadores das metáforas verificadas.

Pelo Quadro 2, nota-se que, dos cinco sentidos, propostos por RudzkaOstyn (2003) para a partícula down, apenas três deles são verificados entre as concordâncias dos $P V S$ selecionados. Isso quer dizer que o Sentido 2 e o Sentido 5 não são tão frequentes no que diz respeito ao uso dessas construções V-P - essa não contemplação de todos os sentidos também ocorre para a partícula up, conforme foi verificado. Mas por quê? Tal ocorrência é discutida nas considerações finais.

\section{Considerações finais}

A principal motivação deste trabalho foi a complexidade semântica dos $P V s$, em especial suas extensões de significado, que afetam diretamente a compreensão e o uso das construções V-P. A partir da investigação realizada, percebeu-se que é possível compreender e explicar os fenômenos que norteiam a construção do significado dos $P V S$ com base na linguística cognitiva em geral, e na semântica cognitiva em particular.

Por questões de delimitação do tema, esta pesquisa tratou exclusivamente dos $P V s$ constituídos pelas partículas up e down, já que estão entre as mais recorrentes no uso da língua inglesa (RUDZKA-OSTYN, 2003). Seus usos frequentes se explicam pela experiência cotidiana (física e abstrata) que se tem em relação a movimentos ou posições para cima e para baixo. Com relação aos sentidos que não foram verificados dentre as concordâncias analisadas, acredita-se que, se a análise fosse estendida para um maior número de concordâncias, tornar-se-ia possível verificar todos os sentidos propostos por Rudzka-Ostyn.

No que diz respeito à idiomaticidade, sabe-se que o fenômeno está relacionado a sentidos figurados, que, na sua maioria, são oriundos de sentidos mais básicos - fator que se reflete nos objetivos específicos estipulados neste trabalho, referentes à identificação das metáforas conceptuais que motivam os sentidos de suas partículas, à averiguação de seus respectivos mapeamentos metafóricos e como esses sentidos expressam esquemas imagéticos. A idiomaticidade é um "prato cheio" para uma análise futura. Ao tratar da idiomaticidade dos $P V s$, não se pode negar o fator da composicionalidade, a qual é variável, apresentando-se em 
diferentes graus, em que o aumento da presença de um implica a diminuição do outro. Esses fenômenos constituem certas estruturas linguísticas que são motivas pelos processos linguísticos, o que vai ao encontro de Kovács (2008?), quando se refere à metaforicidade.

Quanto aos sentidos das partículas, deu-se um destaque considerável ao estudo desenvolvido por Rudzka-Ostyn (2003), uma investigação de grande valia; contudo, a autora, apesar de abordar a importância das metáforas conceptuais no que tange à formação dos $P V s$, não as explora em sua obra. Nesse sentido, a investigação realizada e apresentada no presente artigo, em especial no que diz respeito à verificação dos mapeamentos metafóricos e das metáforas conceptuais relacionadas aos $P V S$ em concordâncias, pode agregar à proposta de caráter pedagógico de Rudzka-Ostyn, de modo que se possa contribuir com a área do ensino/aprendizagem, por exemplo.

No que se refere à TMC, utilizada como base teórica nesta investigação, reiterase que a teoria não trata especificamente de $P V s$ e, além disso, sofre críticas por apresentar algumas lacunas. Porém, a partir deste trabalho, foi possível evidenciar que a TMC, central para esta pesquisa, aliada a outras, aqui consideradas periféricas, conduzem à compreensão dos fenômenos que norteiam os $P V s$, as metáforas conceptuais podem ser verificadas nas construções V-P, já que sua metaforicidade é bastante recorrente, domínios abstratos são entendidos em termos de domínios mais concretos. Essas metáforas verificadas são de caráter orientacional - o que não quer dizer que deixem de apresentar características ontológicas e estruturais, conforme definições de Lakoff e Johnson (1980) e Kövecses (2005). Além disso, destaca-se que, conforme defendido por Lindner (1981), Cappelle (2005), Tyler e Evans (2003) e Rudzka-Ostyn (2003), a contribuição semântica da partícula de um $P V$ tem papel essencial na significação do todo, que, juntamente com o verbo, apresenta forte grau idiomático em muitos casos. 


\title{
Metaphorical motivation on phrasal verbs:
}

\section{a semantic-cognitive conception}

\begin{abstract}
This article aims to show some steps to lead us identify the conceptual metaphors that motivate the senses of "up" and "down" particles, elements in phrasal verbs, as well as ascertain the metaphorical mappings which motivate such metaphors, starting by checking what are the senses that express image schemes proposed by Rudzka-Ostyn (2003). This study is based on Conceptual Metaphor Theory idealized by Lakoff and Johnson (1980), a cognitive semantics approach, in order to think about verbparticle semantic construction, which meanings are metaphoric extended. The Corpus Linguistics is used as a kind of methodological resource since it enables to collect authentic dada to be analysed. From analysis we can verify that "up" and "down" particles are used both prototypical and metaphorical sense, however, the metaphorical one, also known as extended, overwhelm.

Keywords: Phrasal verbs. Cognitive Semantics. Metaphorical motivation. Conceptual Metaphor Theory. Corpus Linguistics.
\end{abstract}

\section{Referências}

BOLINGER, Dwight. The Phrasal Verb in English. Cambridge, Mass.: Harvard University Press, 1971.

CAPPELLE, Bert. Particle Patterns in English: a comprehensive coverage. 2005. $500 \mathrm{f}$. PhD thesis (Doctor in de Taal- en Letterkunde: Germaanse Talen) - Faculteit Letteren, Katholieke Universiteit Leuven, Leuven, 2005. Disponível em: <https:/www.academia.edu/1432972/ Particle_patterns_in_English_A_ comprehensive_coverage $>$. Acesso em: 4 set. 2014.

EVANS, Vyvyan. A Glossary of Cognitive Linguistics. Edinburgh: Edinburgh University Press, 2007.

EVANS, Vyvyan; GREEN, Melanie. Cognitive Linguistics: an introduction. Edinburgh: Edinburgh University Press, 2006.

FRASER, Bruce. The Verb-Particle Combination in English. New York: Academic Press, 1976.

KOVÁCS, Éva. The Traditional vs. Cognitive Approach to English Phrasal Verbs. [S.1.: s. n., 2008?]. Disponível em: <http://www.uni-miskolc. hu/ philos/2011_tom_XVI_1/141.pdf > . Acesso em: 10 dez. 2016. 
KOVÁCS, Éva; SZABÓ, Péter. Idioms: a view from cognitive semantics. Applied Linguistics, [s. 1.], v. 17, n. 3, p. 326-355, 1996. Disponível em: <http:// applij-oxfordjournals-org.ez101.periodicos.capes.gov.br/content/17/3/326.full. pdf + html $>$. Acesso em: 14 jul. 2014.

KÖVECSES, Zoltán. Metaphor in culture: universality and variation. Cambridge: Cambridge University Press, 2005.

LAKOFF, George. Women, Fire, and Dangerous Things. Chicago: The University of Chicago Press, 1990. Original publicado em 1987.

LAKOFF, George; JOHNSON, Mark. Metaphors we live by. Chicago: The University of Chicago Press, 2003. Original publicado em 1980.

LINDNER, Susan. A lexico-semantic analysis with verb-particle constructions with UP and DOWN. 1981. 260 f. Tese (Doutorado) - Universidade da Califórnia, San Diego, 1981.

LIPKA, Leonhard. Semantic Structure and Word-Formation: Verb-Particle Constructions in Contemporary English. München: Fink, 1972. (International Library of General Linguistics, 17.)

Macmillan Phrasal Verbs Plus. Oxford: Macmillan, 2005.

RUDZKA-OSTYN, Brygida. Word Power: phrasal verbs and compounds. Berlin: Mouton de Gruyter, 2003.

SROKA, Kazimierz A. The Syntax of English Phrasal Verbs. The Hague: Mouton, 1972. (Janua Linguarum, Series Practica, 129.)

TYLER, Andrea; EVANS, Vyvyan. The semantics of English prepositions: spatial scenes, embodied meanings and cognition. Cambridge: Cambridge University Press, 2003.

Recebido em 07/01/2016.

Aceito em 05/07/2016. 